\title{
Depth of pleated surfaces in toroidal cusps of hyperbolic 3-manifolds
}

\author{
YING-QING WU
}

\begin{abstract}
Let $F$ be a closed essential surface in a hyperbolic 3-manifold $M$ with a toroidal cusp $N$. The depth of $F$ in $N$ is the maximal distance from points of $F$ in $N$ to the boundary of $N$. It will be shown that if $F$ is an essential pleated surface which is not coannular to the boundary torus of $N$ then the depth of $F$ in $N$ is bounded above by a constant depending only on the genus of $F$. The result is used to show that an immersed closed essential surface in $M$ which is not coannular to the torus boundary components of $M$ will remain essential in the Dehn filling manifold $M(\gamma)$ after excluding $C_{g}$ curves from each torus boundary component of $M$, where $C_{g}$ is a constant depending only on the genus $g$ of the surface.
\end{abstract}

$57 \mathrm{~N} 10$

\section{Introduction}

In this paper a surface $F$ in a 3 -manifold $M$ is a pair $F=(S, \varphi)$, where $S$ is a connected, possibly nonorientable surface, and $\varphi: S \rightarrow M$ is a continuous map which is an immersion almost everywhere. Concepts such as points on $F$ and $\pi_{1}$-injectivity of $F$ are defined in the usual way. See Section 2 for more details.

Let $N$ be a toroidal cusp in a hyperbolic 3-manifold $M$. The depth of a surface $F$ in $N$, denoted by $d_{N}(F)$, is defined as the maximal distance from all points of $F \cap N$ to the boundary torus $T$ of $N$. When $F$ is disjoint from $N$, the depth of $F$ in $N$ is defined as zero. The depth of a closed surface $F$ in $N$ can be arbitrarily large, as one can deform $F$ via homotopy to push $F$ deep into $N$. On the other hand, if $F$ is closed, totally geodesic and has a bounded genus, then the depth of $F$ in $N$ is bounded above by a constant which is independent of $N$ and $M$. See for example Bart [2]. Here it is important to assume that the genus of $F$ is bounded, as the depth of a closed totally geodesic surface in a cusp of the figure 8 knot complement is unbounded. (This follows from a theorem of Leininger [10] and the proof of Theorem 3.1 below.)

We would like to generalize this result to pleated surfaces, which were invented by Thurston [15]. Roughly speaking, a pleated surface is a surface in a hyperbolic 3manifold $M$ which is obtained from a hyperbolic surface by bending along a geodesic 
lamination. Because of that, it shares some properties of totally geodesic surfaces; for example, it has the same area as the underlying hyperbolic surface. However, many important properties of totally geodesic surfaces are not shared by pleated surfaces; for example, a totally geodesic surface in $M$ is always $\pi_{1}$-injective, but that is not true for pleated surfaces.

The following theorem gives an estimation of the depth of closed essential pleated surfaces $F$ in a toroidal cusp $N$ of a hyperbolic manifold $M$. It shows that if $F$ is not coannular to the boundary torus of a toroidal cusp $N$ then its depth in $N$ is bounded above by a constant which depends only on the genus $g$ of $F$.

Theorem 1.1 Let $M$ be a complete hyperbolic 3-manifold, and let $N$ be a toroidal cusp of $M$. Let $F$ be an essential closed pleated surface of genus $g$ in $M$ which is not coannular to the torus $T=\partial N$ in $M$. Then $d_{N}(F)<\ln (2 \pi g)$.

The proof of this theorem will be given in Section 2. One is referred to that section for definitions of cusps and pleated surfaces.

We use the depth estimation of pleated surfaces to study the question of how many Dehn fillings on a toroidal boundary component $T_{0}$ of a compact hyperbolic manifold $M_{0}$ will preserve the $\pi_{1}$-injectivity of an immersed essential surface $F$ in $M_{0}$. A surface $F=(S, \varphi)$ in $M_{0}$ is $\pi_{1}$-injective if $\varphi_{*}: \pi_{1}(S) \rightarrow \pi_{1}\left(M_{0}\right)$ is injective. A closed surface $F$ is essential if it is $\pi_{1}$-injective and is not homotopic to a surface on $\partial M_{0}$. A surface $F$ is coannular to $T_{0}$ if there is a $\pi_{1}$-injective annulus $A$ in $M_{0}$ with one boundary component on each of $F$ and $T_{0}$, in which case the slope of $A \cap T_{0}$ is called a coannular slope of $F$ on $T_{0}$.

The answer to this question is well understood if $F$ is embedded. Denote by $M_{0}(\gamma)$ the manifold obtained from $M_{0}$ by Dehn filling on $T_{0}$ along a slope $\gamma$. It is known that if $F$ is not coannular to $T_{0}$, then $F$ remains essential in $M_{0}(\gamma)$ for all but at most three slopes $\gamma$ by work of the author [17], and if $F$ is coannular to $T_{0}$ with coannular slope $\gamma_{0}$ then $F$ remains essential in $M_{0}(\gamma)$ unless $\Delta\left(\gamma, \gamma_{0}\right) \leq 1$, where $\Delta(\alpha, \beta)$ denotes the geometric intersection number between the two slopes by Culler et al [5, Theorem 2.4.3]. Thus in the latter case $F$ remains essential in $M_{0}(\gamma)$ for all but at most three lines of slopes $\gamma$. Similar results hold for essential laminations and essential branched surfaces. See $\mathrm{Wu}[16]$.

For immersed essential surfaces, the above theorems can be generalized in a weaker sense. Suppose $F$ is an immersed essential surface in a compact hyperbolic manifold $M_{0}$ with $\partial M_{0}=T_{0}$. If $F$ is not coannular to $T_{0}$, then there is a constant $K$ such that $F$ remains essential in $M_{0}(\gamma)$ for all but at most $K$ slopes $\gamma$ by Aitchison and 
Rubinstein [1] and Bart [2]. If $F$ is coannular to $T_{0}$ with coannular slopes $\alpha_{1}, \ldots, \alpha_{n}$, then there is a constant $K$ such that $F$ remains essential in $M_{0}(\gamma)$ when $\Delta\left(\gamma, \alpha_{i}\right)>K$ for all $i$ by $\mathrm{Wu}[18$, Theorem 5.3].

The major difference between the embedded and immersed cases is that in the immersed case there is in general no upper bound for the constant $K$ above. It was shown by the author [18] that for any constant $K$ there is an essential immersed surface $F$ in a hyperbolic manifold $M_{0}$ with a coannular slope $\gamma_{0}$ on $T_{0}$ such that $F$ is compressible in $M_{0}(\gamma)$ for all $\gamma$ satisfying $\Delta\left(\gamma, \gamma_{0}\right) \leq K$. For non-coannular case, Leininger showed in [10] that for any $K$ there is an essential surface in a hyperbolic manifold $M_{0}$ which is totally geodesic (and hence is not coannular to $T_{0}$ ), and is compressible in $M_{0}(\alpha)$ and $M_{0}(\beta)$ with $\Delta(\alpha, \beta)>K$. Thus there is no universal upper bound on the geometric intersection number between "killing slopes" for $F$. It is still an open question whether there is a universal bound for the number of killing slopes for such an $F$ in a hyperbolic manifold $M_{0}$, although we will show that no such upper bound exists if $M_{0}$ is not assumed hyperbolic. See Theorem 3.7 below.

We use the result about the depth of a pleated surface in hyperbolic cusps to study the question of whether there exists an upper bound for the constant $K$ which depends only on the genus of the surface $F$. The following is a simplified version of Corollary 3.2 in the special case that the Dehn filling is performed on a single torus component $T_{0}$ of $\partial M_{0}$.

Theorem 1.2 Let $M_{0}$ be a compact orientable hyperbolic 3-manifold with $T_{0}$ a torus boundary component, and let $F$ be an immersed essential surface in $M_{0}$ which is not coannular to $\partial M_{0}$. Then there is a constant $C_{1}(g)\left(<3200 g^{2}\right)$ depending only on the genus $g$ of $F$, such that $F$ remains essential in $M_{0}(\gamma)$ for all but at most $C_{1}(g)$ slopes $\gamma$ on $T_{0}$.

The theorem shows that an upper bound for the constant $K$ above depending only on the genus of $F$ does exist when $M_{0}$ is hyperbolic and $F$ is not coannular to $T_{0}$. This should be compared with Theorem 3.5, which says that similar bound does not exist in the case that $F$ is coannular to $T_{0}$. It will also be shown (Theorem 3.7) that the condition that $M_{0}$ be hyperbolic is crucial and cannot be removed from the above theorem. A general version of Theorem 1.2 (for Dehn fillings on multiple boundary components of $M_{0}$ ) and its variations are given in Theorem 3.1 and Corollaries 3.2 and 3.3.

The constant $3200 g^{2}$ in Theorem 1.2 is certainly not the best possible. A smaller upper bound in the special case that $F$ is totally geodesic can been found in [2].

Acknowledgement The author was partially supported by NSF grant \#DMS 0203394. 


\section{Proof of Theorem 1.1}

Let $M_{0}$ be a compact, connected, orientable 3-manifold with $T$ a set of tori on $\partial M_{0}$, such that $M=M_{0}-\partial M_{0}$ admits a complete hyperbolic structure. Thus the universal covering space of $M$ is the hyperbolic space $\mathbb{H}^{3}$. We use the upper half space model to identify $\mathbb{H}^{3}$ with

$$
\mathbb{H}^{3}=\{(x, y, z) \mid z>0\}
$$

which is endowed with a Riemannian metric $d s^{2}=\left(1 / z^{2}\right) d r^{2}$, where $d r^{2}$ is the Euclidean metric of $\mathbb{R}^{3}$.

Let $\rho: \mathbb{H}^{3} \rightarrow M$ be the covering map. Recall that a horoball of $\mathbb{H}^{3}$ is a subset isometric to the set $\{(x, y, z) \mid z \geq 1\}$. The boundary of a horoball is called a horosphere. A cusp $N$ of $M$ at a torus boundary component $T_{0}$ of $M_{0}$ is a regular neighborhood of $T_{0}$ in $M_{0}$ with $T_{0}$ removed, such that $\rho^{-1}(N)$ is a set of horoballs in $\mathbb{H}^{3}$ with disjoint interiors. Each component of $\partial M_{0}$ has a unique maximal cusp in $M$, which has the property that there are a pair of horoballs covering it which intersect each other at some point on their boundary. Given a cusp $N$, we may assume that $\rho: \mathbb{H}^{3} \rightarrow M$ has been chosen so that $\widetilde{N}=\{(x, y, z) \mid z \geq 1\}$ is a horoball covering $N$. Let $T=\partial N$, and let $\tilde{T}=\partial \tilde{N}$.

Recall that a surface $F$ in $M$ is a pair $F=(S, \varphi)$, where $S$ is a connected, possibly nonorientable surface, and $\varphi: S \rightarrow M$ is a continuous map which is an immersion almost everywhere. If a surface $F$ in $M$ is $\pi_{1}$-injective then we define the fundamental group of $F$ to be $\pi_{1} F=\varphi_{*}\left(\pi_{1}(S)\right)$. This is well defined up to conjugacy. We can define some other notions for $F$ in the obvious way. For example a point on $F$ is a pair $(x, \varphi)$ with $x \in S$, and a loop on $F$ is the composition of $\varphi$ with a loop on $S$. Thus if $F$ is transverse to a surface $T$ in $M$ then $F \cap T$ is a set of loops in $F$. When there is no confusion, we will also refer to the set $F=\varphi(S)$ as a surface.

A piecewise geodesic arc in a Riemannian manifold $M$ is an $\operatorname{arc} \alpha$ which contains a nowhere dense closed subset $X$ such that the closure of each component of $\alpha-X$ is a geodesic in $M$. We use the definition given by Thurston in [15] for pleated surfaces: A surface $F=(S, \varphi)$ in a hyperbolic manifold $M$ is a pleated surface if $S$ is a complete hyperbolic surface, and $\varphi: S \rightarrow M$ is a continuous map, such that

(1) $\varphi$ is an isometry in the sense that every geodesic segment in $S$ is taken to a piecewise geodesic arc in $M$ which has the same length, and

(2) for each point $x \in S$, there is at least one open geodesic segment $l_{x}$ through $x$ which is mapped to a geodesic segment in $M$. 
The pleating locus of $F$ is the set of points on $S$ which do not have a neighborhood mapped by $\varphi$ to a totally geodesic disk in $M$. We refer the readers to Thurston [15] for some basic properties of pleated surfaces. In particular, it is known that the pleating locus of a pleated surface $(S, \varphi)$ is a geodesic lamination on $S$, which has measure 0 .

Now let $M, N$ and $F$ be as in Theorem 1.1. We may assume that $F=(S, \varphi)$ intersects $N$ nontrivially as otherwise we would have $d_{N}(F)=0$ and hence Theorem 1.1 holds. Fix a point $p_{0}=\varphi\left(s_{0}\right) \in F \cap N$ such that the distance from $p_{0}$ to $T$ equals $d_{N}(F)$. By definition and assumption $S$ is a closed hyperbolic surface of genus $g$, so its universal covering $\widetilde{S}$ is the hyperbolic plane $\mathbb{H}^{2}$. Denote by $\rho_{1}$ the covering map $\rho_{1}: \widetilde{S} \rightarrow S$. Let $\widetilde{s}_{0} \in \widetilde{S}$ be a point with $\rho_{1}\left(\widetilde{s}_{0}\right)=s_{0}$, and let $\widetilde{\varphi}: \widetilde{S} \rightarrow \widetilde{M}$ be a lifting of $\varphi$ such that $\tilde{p}_{0}=\tilde{\varphi}\left(\widetilde{s}_{0}\right)$ is a point in the horoball $\tilde{N}=\{(x, y, z) \mid z \geq 1\}$, and the distance from $\widetilde{p}_{0}$ to the horosphere $\widetilde{T}=\partial \tilde{N}$ equals $d_{N}(F)$. We have the following commutative diagram.

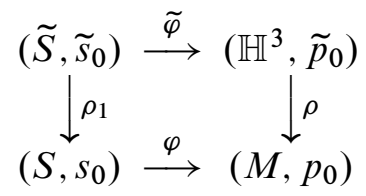

Note that $\widetilde{F}=(\widetilde{S}, \widetilde{\varphi})$ is a pleated surface in $\widetilde{M}$ with pleating locus $\rho_{1}^{-1}(\lambda)$, where $\lambda \subset S$ is the pleating locus of $F$.

Lemma 2.1 Let $\widetilde{T}_{r}=\left\{(x, y, z) \in \mathbb{H}^{3} \mid z=r\right\}$ be a horosphere in $\mathbb{H}^{3}$ at level $r \geq 1$ which intersects $\widetilde{F}$ transversely. Then each component of $\widetilde{F} \cap \widetilde{T}_{r}$ is a circle bounding a disk $D$ in $\widetilde{F}$ lying above $\widetilde{T}_{r}$ in $\mathbb{H}^{3}$.

Proof By assumption $F=(S, \varphi)$ intersects the torus $T_{r}=\rho\left(\widetilde{T}_{r}\right)$ transversely, so $\varphi^{-1}\left(T_{r}\right)$ is a set of circles on $S$. If some circle component $\gamma$ is mapped by $\varphi$ to an essential loop on $T_{r}$ then since $T_{r}$ is $\pi_{1}$-injective in $M, \gamma$ must also be essential on $S$, so $F$ would be coannular to $T_{r}$, contradicting the assumption of Theorem 1.1. Therefore each component of $\varphi^{-1}\left(T_{r}\right)$ is a circle which is trivial on $S$, and hence bounds a disk $D^{\prime}$ on $S$. It follows that $\tilde{\varphi}^{-1}\left(\widetilde{T}_{r}\right)$ is a set of disjoint circles on $\widetilde{S}$, each of which bounds a disk $D$ in $\widetilde{S}$. We need to show that $\tilde{\varphi}(D)$ lies above the horosphere $\widetilde{T}_{r}$.

If this were not true, then since $D$ is compact, there is an interior point $u$ of $D$ such that $\tilde{\varphi}(u)$ has minimal $z$-coordinate in a neighborhood of $\tilde{u}$ in $D$. On the other hand, since $\widetilde{F}=(\widetilde{S}, \widetilde{\varphi})$ is a pleated surface, there is a geodesic segment $\beta$ of $\mathbb{H}^{3}$ lying on $\widetilde{\varphi}(\widetilde{S})$, containing the point $\widetilde{\varphi}(u)$ in its interior. Since a geodesic in $\mathbb{H}^{3}$ is either a Euclidean half circle perpendicular to the $x y$-plane or a vertical line parallel to the $z$-axis, no point of $\beta$ can have locally minimal $z$-coordinate, which is a contradiction. 
Lemma 2.2 Let $R(t)$ be the region on the $\mathbb{H}^{2}$ above the horizontal line at height 1 and inside the Euclidean half circle of radius $t>1$ centered at the origin, as shown in Figure 1.

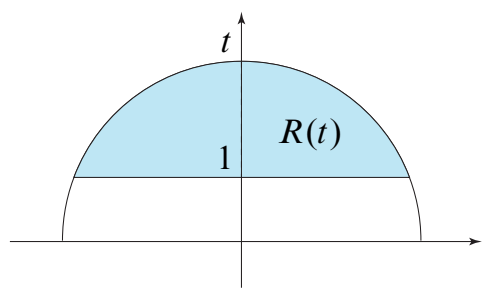

Figure 1

Denote by $A(R(t))$ the area of $R(t)$. Then

$$
A(R(t))=2\left(\sqrt{t^{2}-1}-\arctan \sqrt{t^{2}-1}\right)>2 t-6 .
$$

Proof We have

$$
\begin{aligned}
A(R(t)) & =\iint_{R(t)} \frac{1}{z^{2}} d y d z=2 \int_{1}^{t} \frac{1}{z^{2}} d z \int_{0}^{\sqrt{t^{2}-z^{2}}} d y \\
& =2 \int_{1}^{t} \frac{\sqrt{t^{2}-z^{2}}}{z^{2}} d z \\
& =2\left(\arctan \frac{\sqrt{t^{2}-z^{2}}}{z}-\frac{\sqrt{t^{2}-z^{2}}}{z}\right)_{1}^{t} \\
& =2\left(\sqrt{t^{2}-1}-\arctan \sqrt{t^{2}-1}\right) \\
& >2((t-1)-\pi / 2)>2 t-6 .
\end{aligned}
$$

We identify $\mathbb{H}^{2}$ with the upper half $y z$-plane in $\mathbb{H}^{3}=\mathbb{R}_{+}^{3}$, and denote by $\psi: \mathbb{H}^{3} \rightarrow \mathbb{H}^{2}$ the Euclidean orthogonal projection, ie,

$$
\psi(x, y, z)=(0, y, z) .
$$

Denote by $A(F)$ the area of a surface $F$ in $\mathbb{H}^{3}$. Clearly $\psi$ is area decreasing, ie, $A(\psi(F)) \leq A(F)$ for any surface $F$ in $\mathbb{H}^{3}$.

Lemma 2.3 Let $D$ be a disk in $\tilde{N}$ with $\partial D \subset \widetilde{T}$. Let $\alpha$ be a proper arc on $D$ such that $\alpha^{\prime}=\psi(\alpha)$ is a simple arc on $\mathbb{H}^{2}$. Let $\beta^{\prime}$ be a horizontal arc on $\mathbb{H}^{2}$ joining the two endpoints of $\alpha^{\prime}$, and let $D^{\prime}$ be the disk on $\mathbb{H}^{2}$ bounded by $\alpha^{\prime} \cup \beta^{\prime}$. Then $A(D) \geq 2 A\left(D^{\prime}\right)$. 
Proof Note that $\partial D$ is mapped into the horizontal line at level $z=1$ in $\mathbb{H}^{2}$, hence $\alpha^{\prime} \cup \beta^{\prime}=\partial D^{\prime}$ is a simple closed curve on $\mathbb{H}^{2}$. The arc $\alpha$ cuts $D$ into $D_{1}$ and $D_{2}$. Note that $\psi$ maps $\partial D_{i}$ to a curve on $\mathbb{H}^{2}$ which is the union of $\alpha^{\prime}$ and a path on the horizontal line at $z=1$, hence if $u$ is a point in the interior of $D^{\prime}$ then the winding number of $\psi\left(\partial D_{i}\right)$ around $u$ is \pm 1 . It follows that $u \in \psi\left(D_{i}\right)$, in other words, $\psi\left(D_{i}\right)$ contains $D^{\prime}$. Since $\psi$ is area decreasing, $A\left(D^{\prime}\right) \leq A\left(\psi\left(D_{i}\right)\right) \leq A\left(D_{i}\right)$, and the result follows.

By considering the horosphere with $z$ coordinate $1+\epsilon$ if necessary, we may assume that $\widetilde{F}$ is transverse to the horosphere $\widetilde{T}$ at level $z=1$. Let $D$ be the component of $\widetilde{F} \cap \tilde{N}$ containing the point $\tilde{p}_{0}=\left(x_{0}, y_{0}, z_{0}\right)$. By definition of $\tilde{p}_{0}, z_{0}$ is maximal among $z$-coordinates of all points of $\widetilde{F}$. By Lemma $2.1 \mathrm{D}$ is a disk.

Lemma $2.4 A(D) \geq A\left(R\left(z_{0}\right)\right)>2 z_{0}-6$.

Proof Without loss of generality we may assume that $\tilde{p}_{0}$ is on the $z$-axis, so $\tilde{p}_{0}=$ $\left(0,0, z_{0}\right)$. Recall that $\widetilde{F}$ is a pleated surface. If $\widetilde{p}_{0}$ is on the pleating locus $\tilde{\lambda}$ of $\widetilde{F}$, then it is on a geodesic $\gamma$ of $\mathbb{H}^{3}$ contained in $\widetilde{F}$. If $\tilde{p}_{0}$ is not on the pleating locus $\tilde{\lambda}$, then it lies in the interior of the closure of a component of $\widetilde{F}-\tilde{\lambda}$, which is a totally geodesic surface $P$ in $\mathbb{H}^{3}$ with boundary a disjoint union of geodesics of $\mathbb{H}^{3}$. We will call $P$ an ideal polygon, although it may have infinite area.

First assume that $\tilde{p}_{0}$ is on a geodesic $\gamma$ of $\mathbb{H}^{3}$ such that $\gamma \subset \widetilde{F}$. Rotating $\mathbb{H}^{3}$ along the $z$-axis if necessary, we may assume that $\gamma$ lies in the upper half $y z$-plane, which is a hyperbolic plane $\mathbb{H}^{2}$. Since $\tilde{p}_{0}=\left(0,0, z_{0}\right)$ has maximal $z$-coordinate on $\tilde{F}$ and $\gamma \subset \widetilde{F}$, the curve $\gamma$ is a Euclidean half circle of radius $z_{0}$ on $\mathbb{H}^{2}$ centered at the origin. We now apply Lemma 2.3 to the disk $D$ and the $\operatorname{arc} \alpha=\gamma \cap D$. Note that since $\gamma$ lies on the $y z$-plane, the projection $\alpha^{\prime}=\psi(\alpha)$ equals $\alpha$, which is the part of $\gamma$ lying above the horizontal line $L$ at level $z=1$. Thus the disk $D^{\prime}$ in Lemma 2.3 is exactly the region $R\left(z_{0}\right)$ defined in Lemma 2.2. By Lemma 2.3 we have $A(D) \geq 2 A\left(D^{\prime}\right)=2 A\left(R\left(z_{0}\right)\right)$.

Now assume that $\tilde{p}_{0}$ is in an ideal polygon $P$ of $\mathbb{H}^{3}$ contained in $\widetilde{F}$. Let $q$ be an ideal point of $P$, and let $\gamma$ be a geodesic in $P$ passing through $\tilde{p}_{0}$ and having one ideal endpoint at $q$. Note that at least one component of $\gamma-\tilde{p}_{0}$ lies in $P$, and $\gamma$ is either disjoint from $\partial P$ (and hence contained in $P$ ), or intersects $\partial P$ at a single point. Up to a rotation of $\mathbb{H}^{3}$ along the $z$-axis we may assume that $\gamma$ lies on the $y z$-plane and the ideal point $q$ is on the negative $y$-axis. Let $R^{\prime}$ be the region on $\mathbb{H}^{2}$ lying on the left of the $z$-axis, below $\gamma$ and above the horizontal line $z=1$. Note that $A\left(R^{\prime}\right)=\frac{1}{2} A\left(R\left(z_{0}\right)\right)$. 
If $\gamma \cap \partial P=\varnothing$ then the proof follows as before. So assume $\gamma$ intersects $\partial P$ at a point $u$. Then $u$ lies on a geodesic $\beta$ on $\partial P$, as shown in Figure 2. Let $\beta_{1}$ and $\beta_{2}$ be

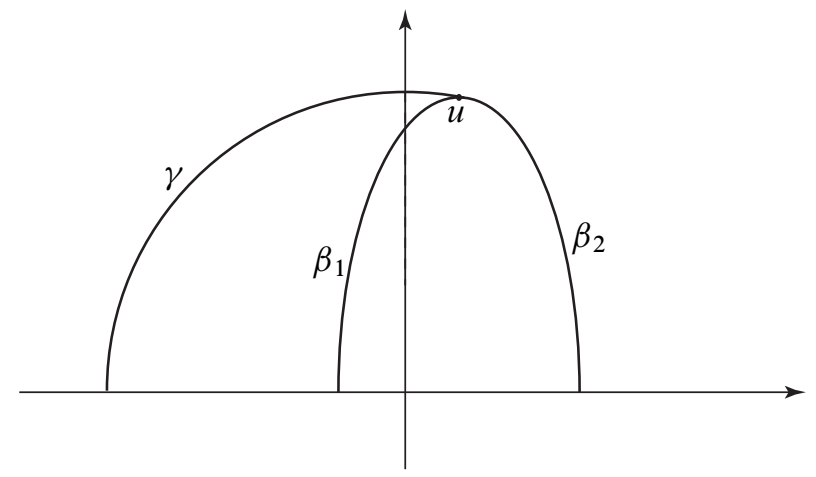

Figure 2

the two components of $\beta-u$, and let $\alpha_{i}$ be the $\operatorname{arc}\left(\gamma \cup \beta_{i}\right) \cap D$. From Figure 2 it is clear that at least one of the projection of $\alpha_{i}$ on $\mathbb{H}^{2}$ is an arc whose union with an arc on the horizontal line at $z=1$ bounds a disk $D^{\prime}$ containing the region $R^{\prime}$ above. By Lemma 2.3 it follows that $A(D) \geq 2 A\left(D^{\prime}\right) \geq 2 A\left(R^{\prime}\right)=A\left(R\left(z_{0}\right)\right)>2 z_{0}-6$.

Proof of Theorem 1.1 By Lemma 2.1 each component of $\varphi^{-1}(N)$ is a disk on the hyperbolic surface $S$. Since $\rho_{1}: \widetilde{S} \rightarrow S$ is a universal covering map, each component of $\rho_{1}^{-1}\left(\varphi^{-1}(N)\right)$ is a disk $D$ which maps homeomorphically by $\rho_{1}$ to a component $D^{\prime}$ of $\varphi^{-1}(N)$; therefore

$$
A(D)=A\left(D^{\prime}\right) \leq A(S)=2 \pi|\chi(S)|=2 \pi(2 g-2) .
$$

Since $\tilde{\varphi}: \widetilde{S} \rightarrow \mathbb{H}^{3}$ is a pleated surface, the area of $\tilde{\varphi}(D)$ equals the area of $D$. Thus by Lemma 2.4 we have

$$
2 \pi(2 g-2) \geq A\left(D^{\prime}\right)=A(D)>2 z_{0}-6,
$$

where $z_{0}$ is the maximum $z$-coordinate of points on $\tilde{\varphi}(\tilde{S})$. Thus $z_{0}<2 \pi g$. Now the hyperbolic distance from a point $p=(x, y, z) \in \mathbb{H}^{3}$ to the horosphere $\widetilde{T}$ at level $z=1$ is $|\ln z|$. Therefore the depth of $F$ in $N$ satisfies $d_{N}(F)=\ln z_{0}<\ln (2 \pi g)$.

\section{Essential surfaces and Dehn filling}

Let $M_{0}$ be a compact orientable hyperbolic 3-manifold with $\partial M_{0}$ a set of tori, and let $M=M_{0}-\partial M_{0}$. A maximal set of cusps in $M$ is a set of cusps $N$ in $M$, one 
for each end of $M$, such that (i) the interiors of the cusps are disjoint, and (ii) $N$ is maximal subject to (i). Note that $N$ may not be unique if $M_{0}$ has more than one boundary components.

Let $N$ be a set of cusps in $M$, one for each end of $M$. Let $T=\partial N$, which is a set of tori in $M$, and is parallel to $\partial M_{0}$ in $M_{0}$. The hyperbolic structure of $M$ induces a Euclidean metric on $T$. The $T$-length $l_{T}(\gamma)$ of a curve $\gamma$ on $\partial M_{0}$ is defined to be the length of a geodesic on $T$ homotopic to $\gamma$ in $M_{0}$. It is known that if $N$ is maximal and $T=\partial N$ then $l_{T}(\gamma) \geq 1$ for any nontrivial curve $\gamma$ on $\partial N$. See Bleiler and Hodgson [3].

A multiple slope is a set of essential simple closed curves $\gamma=\left(\gamma_{1}, \ldots, \gamma_{k}\right)$, such that there is at most one $\gamma_{i}$ for each boundary component of $M_{0}$. Define $M_{0}(\gamma)$ to be the manifold obtained by attaching $k$ solid tori to $M_{0}$ such that $\gamma_{i}$ bounds a meridian disk in the $i$-th attached solid torus.

Theorem 3.1 Let $F=(S, \varphi)$ be a closed $\pi_{1}$-injective surface of genus $g$ in a compact, orientable, hyperbolic 3-manifold $M_{0}$ with $\partial M_{0}$ a set of tori, and assume that $F$ is not coannular to $\partial M_{0}$. Then there is a constant $C_{0}(g)<4 \pi^{2} g$ depending only on $g$, such that if $N$ is a maximal set of cusps of $M=M_{0}-\partial M_{0}$, and $\gamma=\gamma_{1} \cup \cdots \cup \gamma_{k}$ is a multiple slope on $\partial M_{0}$ such that $l_{T}\left(\gamma_{i}\right)>C_{0}(g)$ for all $i$, then $F$ remains $\pi_{1}-$ injective in $M_{0}(\gamma)$.

Proof This follows from Theorem 1.1 and a theorem of Bart [2, Theorem 1.1], which says $F$ remains essential in $M(\gamma)$ if one can choose a set of cusps disjoint from the projection of the convex hull of the limit set of $\pi_{1}(F)$, and the length of each $\gamma_{i}$ is greater than $2 \pi$. We give a proof for the convenience of the readers.

By assumption $M=M_{0}-\partial M_{0}$ admits a complete hyperbolic structure of finite volume. Recall that the group of the surface $F=(S, \varphi)$ is defined to be the subgroup $G=\varphi_{*}\left(\pi_{1}(S)\right)$ of $\pi_{1}(M)$. By Thurston [14], either $F$ is a virtual fiber, or $G$ is geometrically finite; but since $F$ is closed while $M$ is noncompact, $F$ cannot be a virtual fiber. Hence it must be geometrically finite.

Let $H(G)$ be the convex hull of the limit set of $G$ in $\mathbb{H}^{3}$. Consider the covering space $M_{1}$ of $M$ corresponding to the subgroup $G$. Let $\rho^{\prime}: \mathbb{H}^{3} \rightarrow M_{1}$ and $\rho^{\prime \prime}: M_{1} \rightarrow M$ be the covering maps. Then $\rho^{\prime}(H(G))$ is a compact set in $M_{1}$ homeomorphic to $S \times I$ (or $S$ if $G$ is Fuchsian), and hence $\rho(H(G))=\rho^{\prime \prime}\left(\rho^{\prime}(H(G))\right.$ ) is compact in $M$. See Morgan [12]. The boundary of $\rho^{\prime}(H(G))$ consists of two pleated surfaces $F_{1}^{\prime}, F_{2}^{\prime}$ in $M_{1}$ ( $F_{1}^{\prime}=F_{2}^{\prime}$ if $G$ is Fuchsian), which map to pleated surfaces $F_{1}, F_{2}$ in $M$ by $\rho^{\prime \prime}$. Since $\rho: \mathbb{H}^{3} \rightarrow M$ is a covering map, its restriction on $H(G)$ is 
an immersion; hence the frontier of $\rho(H(G))$ is contained in $\rho(\partial H(G))=F_{1} \cup F_{2}$. By Theorem 1.1, the depth of $F_{i}$ in a cusp of $M$ is bounded by $\ln (2 \pi g)$, so the depth of $\rho(H(G))$ in $N$ is also bounded by $\ln (2 \pi g)$.

For simplicity of notation let us assume $k=1$. The proof for the general case is similar. Thus $N$ is a cusp adjacent to a torus component $T_{0}$ of $\partial M_{0}$ containing the Dehn filling slope $\gamma$, and the length of $\gamma$ on $T=\partial N$ is greater than $4 g \pi^{2}$. Let $T^{\prime}$ be the Euclidean torus in the cusp $N$ at depth $\ln (2 \pi g)$. By lifting to the universal covering space $\mathbb{H}^{3}$ one can see that the length of a geodesic curve $\gamma^{\prime}$ on $T^{\prime}$ isotopic to $\gamma$ in $N$ is greater than $\left(4 g \pi^{2}\right) /(2 \pi g)=2 \pi$.

Denote by $M^{\prime}$ the manifold obtained from $M$ by cutting off the cusp bounded by $T^{\prime}$. By the $2 \pi$ theorem of Gromov-Thurston [8; 3], if $\gamma$ is a slope on $T^{\prime}$ with geodesic length greater then $2 \pi$ then the hyperbolic structure on $M^{\prime}$ can be extended over the Dehn filling solid torus to obtain a negatively curved metric on the Dehn filled manifold $M^{\prime}(\gamma)$, which is clearly homeomorphic to $M_{0}(\gamma)$ by a homeomorphism rel $\rho(H(G))$. Up to homotopy we may assume that $F$ is in $\rho(H(G))$. If $\alpha$ is an essential closed curve on $S$, then $\varphi(\alpha)$ is homotopic to a geodesic $\alpha^{\prime}$ in $M$, which lifts to a geodesic in the convex hull $H(G)$, and hence $\alpha^{\prime}$ lies in $\rho(H(G))$, which is a subset of $M^{\prime}$. It follows that $\alpha^{\prime}$ remains a geodesic in $M^{\prime}(\gamma)$, which must be essential because $M^{\prime}(\gamma)$ is negatively curved. Therefore $F=(S, \varphi)$ is $\pi_{1}$-injective in $M^{\prime}(\gamma)$, and hence in $M_{0}(\gamma)$.

Given a Euclidean torus $T$, there are only finitely many curves of length at most $t$. In fact, there is a constant $C(t)$, independent of $T$, such that if the minimum length of closed geodesics on $T$ is 1 then there are at most $C(t)$ simple closed geodesics $\gamma$ on $T$ with $l_{T}(\gamma) \leq t$. Using the idea in the proof of [3, Lemma 12] one can show that

$$
C(t) \leq \frac{(\pi / 2)(t+1 / 2)^{2}}{\sqrt{3} / 2}+1
$$

so for $t \geq 4 \pi^{2}$ we have $C(t)<2 t^{2}$. The following result is now an immediate corollary of Theorem 3.1, as it is known that the minimal length of a closed geodesic on the boundary of a maximal set of cusps is at least 1 [3].

Corollary 3.2 Let $M_{0}$ be a compact orientable hyperbolic 3-manifold with toroidal boundary, and let $F$ be an immersed essential surface of genus $g$ in $M_{0}$ which is not coannular to $\partial M_{0}$. Then after excluding at most $C\left(4 \pi^{2} g\right)\left(<3200 g^{2}\right)$ curves on each component of $\partial M_{0}, F$ remains essential in all $M(\gamma)$.

Denote by $\Delta\left(\gamma_{1}, \gamma_{2}\right)$ the minimal geometric intersection number between two slopes $\gamma_{1}$ and $\gamma_{2}$ on a torus $T$. There is another constant $C^{\prime}(t)$ such that if (i) $T$ is a 
Euclidean torus such that the minimal length of closed geodesics on $T$ is at least 1 , and (ii) $\gamma_{1}$ and $\gamma_{2}$ are closed geodesics of length at most $t$ on $T$, then $\Delta\left(\gamma_{1}, \gamma_{2}\right)<C^{\prime}(t)$. Using the idea in the proof of [3, Theorem 14] one can show that

$$
C^{\prime}(t)<\frac{2}{\sqrt{3}} t^{2}<2 t^{2}
$$

Corollary 3.3 Let $M_{0}$ be a compact orientable hyperbolic 3-manifold with toroidal boundary, and let $F$ be an immersed essential surface of genus $g$ in $M_{0}$ which is not coannular to $\partial M_{0}$. Let $\gamma_{1}, \gamma_{2}$ be slopes on a torus component $T_{0}$ of $\partial M_{0}$. If $F$ is compressible in both $M_{0}\left(\gamma_{1}\right)$ and $M_{0}\left(\gamma_{2}\right)$, then $\Delta\left(\gamma_{1}, \gamma_{2}\right)<C^{\prime}\left(4 \pi^{2} g\right)<3200 g^{2}$.

This should be compared with Leininger's result [10], which says that there is no universal bound for $\Delta\left(\gamma_{1}, \gamma_{2}\right)$ if there is no constraint about the genus of $F$.

In Theorem 3.1 we assumed that the manifold $M_{0}$ is hyperbolic, and the surface $F$ is not coannular to $\partial M_{0}$. Neither condition can be removed. The following theorem is part of [18, Theorem 1.1].

Theorem 3.4 Let $M$ be a compact, orientable, hyperbolic 3-manifold, and let $T$ be a torus component of $\partial M$. Let $F$ be a closed essential surface in $M$.

(1) If $F$ is coannular to $T$ with coannular slopes $\gamma_{1}, \ldots, \gamma_{n}$, then there is an integer $K$ such that $F$ remains $\pi_{1}$-injective in $M(\gamma)$ for all $\gamma$ satisfying $\Delta\left(\gamma, \gamma_{i}\right) \geq K$, $i=1, \ldots, n$.

(2) There is no universal bound on the constant $K$ in (1): For any constant $C$, there is a closed essential surface $F$ in a hyperbolic manifold $M$ with coannular slope $\beta$ on $T=\partial M$, such that $F$ is not $\pi_{1}$-injective in $M(\gamma)$ for all $\gamma$ with $\Delta(\gamma, \beta) \leq C$.

Part (1) of the theorem is a finiteness theorem, while part (2) shows there is no upper bound in general for the number of lines of surgeries which will kill the essential surface. The genera of the surfaces used in the proof of part (2) increase as $C$ increases. Because of Theorem 3.1, one may wonder if a similar theorem would be true for surfaces coannular to $\partial M$. More precisely, does there exist a constant $C(g)$ depending only on the genus of $F$, such that $F$ remains essential in $M(\gamma)$ for all but at most $C(g)$ lines of slopes $\gamma$ ? The following theorem shows that no such constant $C(g)$ exist. Recall that with respect to the standard meridian-longitude pair $(m, l)$ of a knot $K$ in $S^{3}$, a slope $\gamma=p m+q l$ on $\partial N(K)$ is represented by a rational number $p / q$ or $1 / 0$. See Rolfsen [13]. Thus $M(\gamma)=M(p / q)$. 
Theorem 3.5 Let $K$ be a nonfibered hyperbolic knot in $S^{3}$ with genus $g$, and let $M=S^{3}-$ Int $N(K)$ be the knot exterior. Then for any constant $C$ there is an immersed essential surface $F$ of genus $2 g$ with the longitude of $K$ as a coannular slope, such that $F$ is compressible in $M(\gamma)$ for all $\gamma=p / q$ satisfying $0<p \leq C$.

Proof Let $S_{1}$ be a minimal Seifert surface of $K$ in $M$. Let $S$ be the double of $S_{1}$, which is an abstract surface obtained by taking two copies of $S_{1}$ joined by an annulus $A$. Let $\varphi_{k}: S \rightarrow M$ be the map which sends the two copies of $S_{1}$ to $S_{1}$, and $A$ to an annulus wrapping $k$ times around the torus $T=\partial M$. The surface $F_{k}=\left(S, \varphi_{k}\right)$ is called a Freedman tubing of $S_{1}$ (with wrapping number $k$ ). See Freedman and Freedman [6], Cooper and Long [4] and Wu [18] for more details. Since $K$ is hyperbolic and not fibred, by a theorem of Freedman and Freedman [6], Cooper and Long [4] and Li [11], $F_{k}$ is $\pi_{1}$-injective in $M$ when $k$ is sufficiently large. See Wu [18] for an alternative proof.

Lemma 3.6 If $k$ is a multiple of $p$ then the surface $F_{k}$ is compressible in $M(p / q)$.

Proof Put $k=p r$. Let $\alpha$ be an essential arc on the annulus $\varphi_{k}(A)$ which wraps $k$ times around the meridian $m$ and 0 times along the longitude $l$. Then $\alpha \cdot\left(l^{q r}\right)$ represents $k m+(q r) l=r(p m+q l)$ in $\pi_{1}(T)$, and hence is null-homotopic in the Dehn filling solid torus $V$. Thus $\alpha$ is homotopic to $l^{-q r}$ in $V$, so the annulus $\varphi_{k}(A)$ is rel $\partial A$ homotopic to a degenerate annulus with image on the boundary of the Seifert surface $S_{1}$. Therefore the immersed surface $F_{k}$ is homotopic in $M(p / q)$ to an embedding of $S$ in $M$ with image $\partial N\left(S_{1}\right)$, the boundary of a regular neighborhood of $S_{1}$. Since $N\left(S_{1}\right)$ is a handlebody, $\partial N\left(S_{1}\right)$ is compressible in $M$, hence $\varphi_{k}$ is also compressible in $M(p / q)$.

Now given any positive integer $C$, let $k$ be a positive integer which is a multiple of all $p$ with $0<p \leq C$. We may assume that $C$ is sufficiently large, so the surface $F_{k}$ is essential by the Freedman-Cooper-Long Theorem. By Lemma 3.6, the surface $F_{k}$ is compressible in $M(p / q)$ for all $p / q$ with $0<p \leq C$. This completes the proof of Theorem 3.5.

The following theorem shows that Theorem 3.1 is false if the manifold $M$ is not assumed to be hyperbolic.

Theorem 3.7 There is a nonhyperbolic compact orientable irreducible 3-manifold $M$ with $\partial M=T$ a torus, a fixed closed connected orientable surface $S$ of positive genus, and an immersion $\varphi_{n(k)}: S \rightarrow M$ for any positive integer $k$, such that the essential surfaces $F_{k}=\left(S, \varphi_{n(k)}\right)$ satisfy the following conditions. 
(i) $F_{k}$ is $\pi_{1}$-injective.

(ii) $F_{k}$ is not coannular to $T$.

(iii) There are $k$ distinct slopes $\gamma_{1}, \ldots, \gamma_{k}$ on $T$ such that $F_{k}$ is compressible in $M\left(\gamma_{i}\right)$ for $i=1, \ldots, k$.

Proof Let $K_{1}$ be a hyperbolic knot in $S^{3}$ which is not a fibred knot. Let $K=$ $C_{p, q}\left(K_{1}\right)$ be a $(p, q)$ cable knot of $K_{1}$, and let $M=S^{3}-\operatorname{Int} N(K)$ be the exterior of $K$. Denote by $V=N\left(K_{1}\right)$ a regular neighborhood of $K_{1}$ which contains $K$ as a cable knot. Let $M_{1}=S^{3}-$ Int $V$, and let $X=V-\operatorname{Int} N(K)$. Let $T_{1}=\partial M_{1}$. Thus $M=M_{1} \cup_{T_{1}} X$.

To construct the immersed surfaces $F_{k}$, let $S_{1}$ be a minimal genus Seifert surface of the hyperbolic knot $K_{1}$, and let $\varphi_{n}: S \rightarrow M_{1}$ be a Freedman tubing of two copies of $S_{1}$ with wrapping number $n$. By the proof of Theorem 3.5 we may choose $n(k)$ so that (a) $F_{k}=\left(S, \varphi_{n(k)}\right)$ is $\pi_{1}$-injective, and (b) $F_{k}$ is compressible in $M_{1}(\gamma)$ for all $\gamma=r / s$ satisfying $1 \leq r \leq k p q+1$. In particular, $F_{k}$ satisfies condition (i).

Let $\gamma_{i}=(i p q+1) / i$. By a theorem of Gordon [7, Corollary 7.3], we have

$$
M\left(\gamma_{i}\right)=M\left(\frac{i p q+1}{i}\right)=M_{1}\left(\frac{i p q+1}{i q^{2}}\right) .
$$

By definition, $F_{k}$ is compressible in $M_{1}\left((i p q+1) / i q^{2}\right)$ for all $i$ satisfying $1 \leq i \leq k$. Therefore $F_{k}$ satisfies condition (iii). It remains to show that $F_{k}$ is not coannular to $T$.

First notice that the only coannular slope of $F_{k}$ in the manifold $M_{1}$ is the longitude. This is because $F_{k}$ lifts to a closed surface in the universal abelian covering of $M_{1}$, so any closed curve on $F_{k}$ lifts, but the longitude is the only primitive closed curve on $T_{1}=\partial M_{1}$ that lifts.

Now suppose $A$ is an essential annulus in $M$ with one boundary component in each of $F$ and $T$. By a homotopy we may assume that $A$ is transverse to $T_{1}$, and the number of components of $A \cap T_{1}$ is minimal. Since $T_{1}$ is $\pi_{1}$-injective in $M$, each component of $A \cap M_{1}$ and $A \cap X$ is an essential annulus in $M_{1}$ or $X$, except the one which has a boundary component on $F$, denoted by $A_{1}$. By the above, $\beta=A_{1} \cap T_{1}$ is a longitude of $K_{1}$. On the other hand, the component $A_{2}$ of $A \cap X$ which has $\beta$ as a boundary component is an essential annulus in $X$, and it is well known that such an annulus is isotopic to a union of fibers [9, Theorem VI.34]; in particular, the longitude $\beta$ of $K_{1}$ is a fiber of $X$. This is a contradiction because after trivial filling along the meridional slope $m$ on $T$ the Seifert fibration on $X$ extends to a Seifert fibration of the solid torus $X(m)$ containing $K$ as a regular fiber, so a fiber on $T_{1}$ has slope $p / q$. 


\section{References}

[1] I R Aitchison, JH Rubinstein, Incompressible surfaces and the topology of 3dimensional manifolds, J. Austral. Math. Soc. Ser. A 55 (1993) 1-22 MR1231691

[2] A Bart, Surface groups in some surgered manifolds, Topology 40 (2001) 197-211 MR1791272

[3] S A Bleiler, C D Hodgson, Spherical space forms and Dehn filling, Topology 35 (1996) 809-833 MR1396779

[4] D Cooper, D D Long, Virtually Haken Dehn-filling, J. Differential Geom. 52 (1999) 173-187 MR1743462

[5] M Culler, C M Gordon, J Luecke, P B Shalen, Dehn surgery on knots, Ann. of Math. (2) 125 (1987) 237-300 MR881270

[6] B Freedman, M H Freedman, Kneser-Haken finiteness for bounded 3-manifolds locally free groups, and cyclic covers, Topology 37 (1998) 133-147 MR1480882

[7] C M Gordon, Dehn surgery and satellite knots, Trans. Amer. Math. Soc. 275 (1983) 687-708 MR682725

[8] M Gromov, W Thurston, Pinching constants for hyperbolic manifolds, Invent. Math. 89 (1987) 1-12 MR892185

[9] W Jaco, Lectures on three-manifold topology, CBMS Regional Conference Series in Math. 43, Amer. Math. Soc. (1980) MR565450

[10] C J Leininger, Compressing totally geodesic surfaces, Topology Appl. 118 (2002) 309-328 MR1874553

[11] T Li, Immersed essential surfaces in hyperbolic 3-manifolds, Comm. Anal. Geom. 10 (2002) 275-290 MR1900752

[12] J W Morgan, On Thurston's uniformization theorem for three-dimensional manifolds, from: “The Smith conjecture (New York, 1979)", (J W Morgan, H Bass, editors), Pure Appl. Math. 112, Academic Press, Orlando, FL (1984) 37-125 MR758464

[13] D Rolfsen, Knots and links, Math. Lecture Series 7, Publish or Perish, Houston, TX (1990) MR1277811

[14] W P Thurston, The geometry and topology of three-manifolds, Princeton Univ. Math. Dept. Lecture Notes (1979) Available at http://msri.org/publications/books/ gt $3 \mathrm{~m} /$

[15] W P Thurston, Hyperbolic structures on 3-manifolds. I. Deformation of acylindrical manifolds, Ann. of Math. (2) 124 (1986) 203-246 MR855294

[16] Y Q Wu, Essential laminations in surgered 3-manifolds, Proc. Amer. Math. Soc. 115 (1992) 245-249 MR1104405

[17] Y Q Wu, Incompressibility of surfaces in surgered 3-manifolds, Topology 31 (1992) 271-279 MR1167169 
[18] Y Q Wu, Immersed essential surfaces and Dehn surgery, Topology 43 (2004) 319-342 MR2052966

Department of Mathematics, University of Iowa Iowa City, IA 52242

wu@math. uiowa.edu

Received: 10 March 2009 